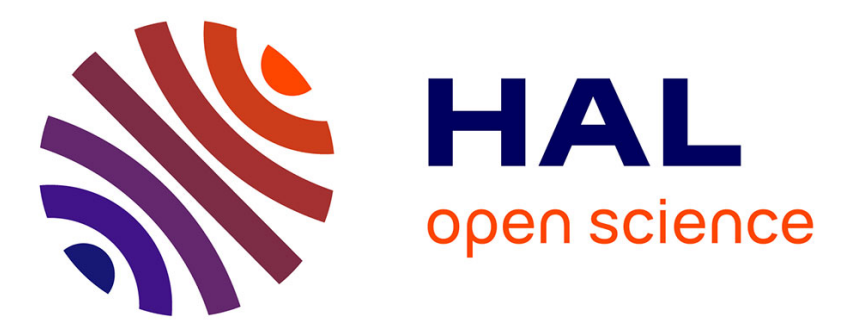

\title{
Dopage béryllium de couches InGaAs élaborées par épitaxie jet moléculaire: étude de la compensation
}

A. Roizes, J.P. David, A. Lecorre, J. Caulet, M. Gauneau, S. Loualiche, H. L'Haridon, D. Lecrosnier

\section{- To cite this version:}

A. Roizes, J.P. David, A. Lecorre, J. Caulet, M. Gauneau, et al.. Dopage béryllium de couches InGaAs élaborées par épitaxie jet moléculaire: étude de la compensation. Revue de Physique Appliquée, 1989, 24 (4), pp.447-451. 10.1051/rphysap:01989002404044700 • jpa-00246066

\section{HAL Id: jpa-00246066 https://hal.science/jpa-00246066}

Submitted on 1 Jan 1989

HAL is a multi-disciplinary open access archive for the deposit and dissemination of scientific research documents, whether they are published or not. The documents may come from teaching and research institutions in France or abroad, or from public or private research centers.
L'archive ouverte pluridisciplinaire HAL, est destinée au dépôt et à la diffusion de documents scientifiques de niveau recherche, publiés ou non, émanant des établissements d'enseignement et de recherche français ou étrangers, des laboratoires publics ou privés. 
Classification

Physics Abstracts

$72.15 \mathrm{G}-72.80 \mathrm{E}-73.90$

\title{
Dopage béryllium de couches InGaAs élaborées par épitaxie jet moléculaire : étude de la compensation
}

\author{
A. Roizes $\left({ }^{1}\right)$, J. P. David $\left({ }^{1}\right)$, A. Lecorre $\left({ }^{2}\right)$, J. Caulet $\left({ }^{2}\right)$, M. Gauneau $\left({ }^{2}\right)$, S. Loualiche $\left({ }^{2}\right)$, H. \\ L'Haridon ( ${ }^{2}$ ) et D. Lecrosnier ( ${ }^{2}$ ) \\ (1) ONERA, CERT, DERTS, B.P. 4025, 31055 Toulouse Cedex, France \\ ( ${ }^{2}$ CNET, Lannion B, route de Trégastel, 22301 Lannion, France
}

(Reçu le $1^{\text {er }}$ juillet 1988, révisé et accepté le 12 décembre 1988)

\begin{abstract}
Résumé. - Des couches épitaxiales $\mathrm{In}_{x} \mathrm{Ga}_{1-x} \mathrm{As}$, élaborées en accord paramétrique sur substrat InP par la technique d'épitaxie par jets moléculaires ont été dopées avec du béryllium de $10^{16}$ à $5 \times 10^{19} \mathrm{at} / \mathrm{cm}^{3}$. D'après les mesures électriques, les couches de type $\mathrm{P}$ sont fortement compensées et à faible niveau de dopage la conductivité peut être de type $\mathrm{N}$. Les analyses d'impuretés par sonde ionique montrent que l'oxygène est responsable de ce comportement. Les mesures d'effet Hall, en fonction de la température, ont montré que suivant le niveau d'oxygène, il pouvait exister une zone $\mathrm{N}$ en surface ou à l'interface avec le substrat InP semiisolant, responsable des faibles mobilités de Hall mesurées.
\end{abstract}

\begin{abstract}
Be doped $\operatorname{In}_{x} \mathrm{Ga}_{1-x}$ As epitaxial layers, with concentration level from $10^{16}$ to $5 \times 10^{19}$ at $/ \mathrm{cm}^{3}$, have been grown lattice matched on InP substrate by molecular beam epitaxy. Room temperature Hall effect measurements, indicate low mobility for $\mathrm{P}$ type samples while for low Be doping the conduction can be $\mathrm{N}$ type. Secondary Ion Mass Spectroscopy analysis shows that the electrical behavior is linked to the oxygen to beryllium concentration ratio in the layer. The temperature Hall effect measurements show that a surface or an interface $\mathbf{N}$ type layer can be created, depending on the oxygen to beryllium ratio concentration and is responsible for the apparent low Hall mobilities.
\end{abstract}

\section{Introduction.}

Les propriétés électriques et optiques du matériau ternaire $\operatorname{In}_{x} \mathrm{Ga}_{1-x} \mathrm{As}$, permettent d'envisager la réalisation de fonctions optoélectroniques intégrées sur substrat InP. La fabrication de ces dispositifs nécessite la maîtrise du dopage des différentes couches constituant les composants individuels. Dans la technique d'épitaxie par jets moléculaires, pour le dopage de type $P$, parmi les impuretés acceptrices possibles $(\mathrm{Zn}, \mathrm{Mg}, \mathrm{Cd}, \mathrm{Be})$ le béryllium est choisi du fait d'un meilleur coefficient de collage. Cependant, une compensation est souvent observée dans les couches de type $\mathrm{P}$, quelle que soit d'ailleurs la technique d'épitaxie, et il est difficile de maîtriser de manière reproductible des dopages inférieurs à $5.10^{17} \mathrm{~cm}^{3}$ [2-4].

Dans cette étude, nous avons cherché à expliquer les mécanismes de compensation de ces couches à partir de mesures d'effet Hall et de SIMS (Secondary Ion Mass Spectrometry).
2. Caractéristiques électriques des couches à $300 \mathrm{~K}$.

La figure 1 représente les variations de la concentration du dopant évaluée par SIMS et de la densité de porteurs libres à $300 \mathrm{~K}$, évaluée à partir de la constante de Hall, en fonction de la température de la source de béryllium. Les résultats obtenus montrent que les différents échantillons se divisent en deux catégories suivant le type de conductivité au sens de l'effet Hall :

Pour les uns la conductivité des couches est assurée par des trous comme normalement attendu avec cependant une mobilité des porteurs bien inférieure à celle mesurée sur des échantillons VPE (Vapor Phase Epitaxy) ayant des densités de porteurs identiques [2] (Fig. 2) ;

Pour les autres la conductivité est de type $\mathrm{N}$, ils correspondent le plus souvent à des concentrations de béryllium inférieures à $10^{18} \mathrm{at} / \mathrm{cm}^{3}$.

Les analyses SIMS des impuretés dans les couches ont montré que l'oxygène est l'impureté principale 


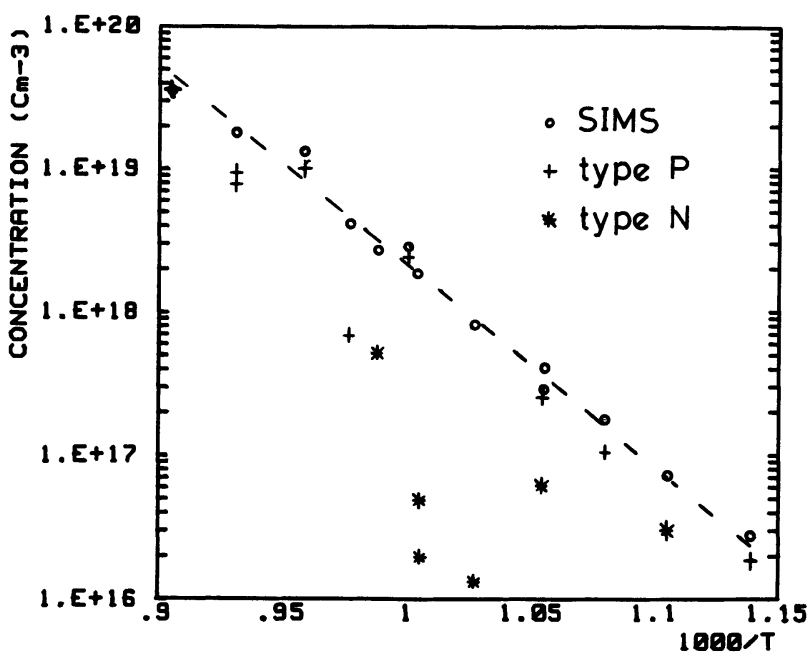

Fig. 1. - Concentration de porteurs libres, déterminée par effet Hall et concentration de béryllium mesurée par SIMS en fonction de l'inverse de la température de la source de béryllium.

[Hall effect carriers concentration and SIMS beryllium concentration as function of $\mathrm{Be}$ effusion cell reciprocal temperature.]

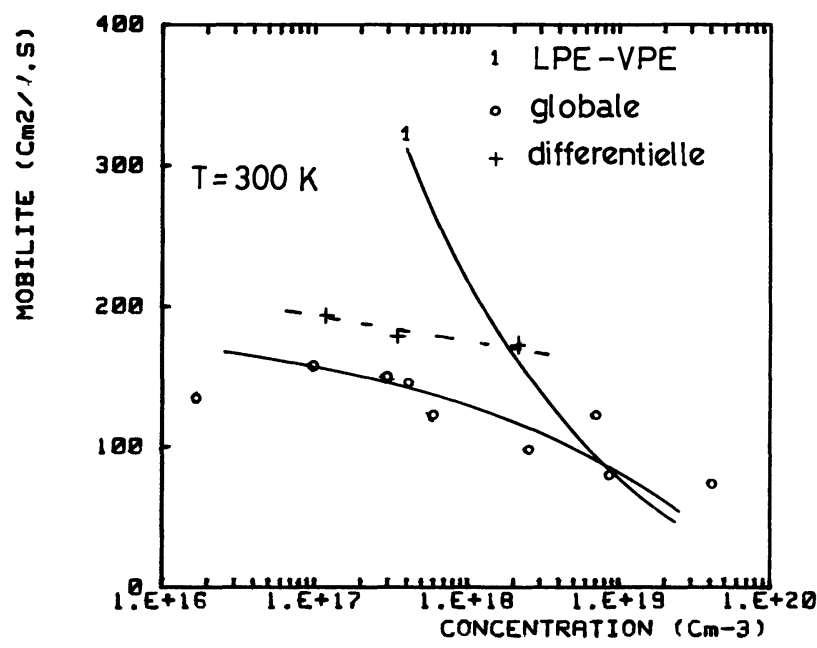

Fig. 2. - Mobilité de Hall d'échantillons de type $\mathrm{P}$ à $300 \mathrm{~K}$ en fonction de la concentration d'impuretés : (O) Mobilité moyenne des couches $\mathrm{MBE}$ de type $\mathrm{P}$, dopées $\mathrm{Be}$, (+) Mobilité de Hall différentielle, (1) Mobilité de Hall d'échantillons de type P (VPE/LPE) (Réf. [2]).

[Hall mobilities of $P$ type doped layers as function of ionised impurity concentration: (O) mean mobilities of MBE Be doped layers ; (+) differential Hall mobilities ; (1) mobilities of VPE/LPE samples from reference [2].]

de contamination et sa densité croît avec la concentration de béryllium. Les figures $3 a$ et $3 b$ montrent qu'il existe un renforcement de la densité d'oxygène à l'interface et à la surface de la couche. Les échantillons de type $\mathrm{N}$ ont une concentration d'oxygène supérieure à celle du béryllium (Fig. 4) et

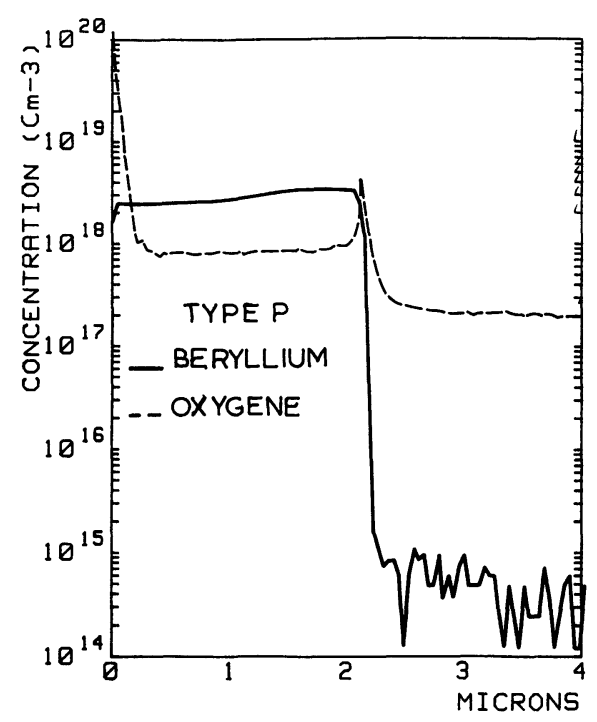

a)

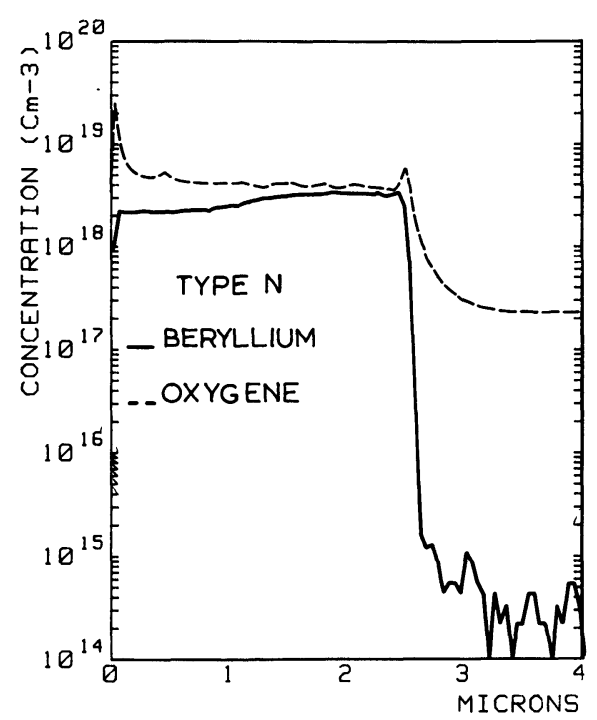

b)

Fig. 3. - Profils typiques de répartition d'oxygène et de béryllium déterminés par SIMS : a) échantillon type $P$ au sens de l'effet Hall; b) échantillon type $\mathrm{N}$ au sens de l'effet Hall.

[Typical SIMS profiles of beryllium and oxygen : a) $P$ type Hall mobility sample ; b) $\mathrm{N}$ type Hall mobility sample.]

il existe une forte différence de concentration en oxygène des échantillons de type $\mathrm{N}$ et ceux de type $\mathrm{P}$ ayant une concentration équivalente de béryllium.

Cette constatation confirme l'hypothèse émise par Stall et al., concernant le caractère donneur de l'oxygène dans $\operatorname{In}_{x} \mathrm{Ga}_{1-x}$ As [1].

\section{Etude de la compensation des couches.}

Des mesures d'effet Hall ont été réalisées entre la température de l'azote liquide et $500 \mathrm{~K}$ : les échantillons de type $\mathrm{N}$ à $77 \mathrm{~K}$ le sont également sur tout le 


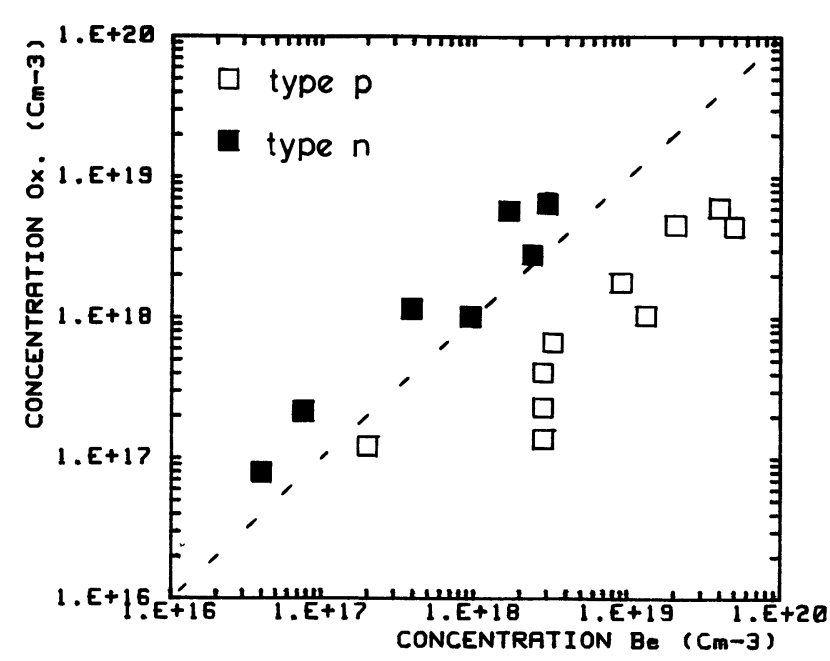

Fig. 4. - Concentration d'oxygène en fonction de la concentration de béryllium, déterminées par SIMS.

[SIMS oxygen concentration versus beryllium concentration.]

domaine de température ; par contre les échantillons de type $\mathrm{P}$ à $77 \mathrm{~K}$, ayant un dopage de béryllium inférieur à $10^{18} \mathrm{at} / \mathrm{cm}^{3}$, changent de type de conductivité, au sens de l'effet Hall, au-dessus de $250 \mathrm{~K}$. La température de transition est d'autant plus élevée que la concentration de béryllium est élevée par rapport à celle de l'oxygène.

Pour ces derniers échantillons, un tel comportement s'explique a priori par une conductivité mixte d'électrons et de trous.

La température d'inversion du type de conductivité étant éloignée de $77 \mathrm{~K}$, nous avons choisi d'évaluer la densité d'impuretés ionisées à partir des mesures de mobilité à cette température. Pour cela nous avons utilisé les résultats de Pearsall et al. [2], qui indiquent la dépendance de la mobilité des électrons et des trous avec la densité totale d'impuretés ionisées. Ce résultat, joint à l'évaluation de la densité de porteurs libres, permet d'en déduire les concentrations de centres électriquement actifs de chaque échantillon.

Les densités d'accepteurs électriquement actifs, déterminées par cette méthode sont supérieures aux concentrations de béryllium déterminées par SIMS : l'écart est d'autant plus important que la densité de béryllium dans l'échantillon est faible, il est moindre pour les échantillons les plus dopés (Fig. 5). Les erreurs expérimentales SIMS ne peuvent pas expliquer un tel écart.

D'autre part l'hypothèse d'une conductivité mixte, conduit à une évaluation de la concentration d'électrons de la couche incompatible avec la concentration intrinsèque de porteurs de $\mathrm{In}_{x} \mathrm{Ga}_{1_{-}}$As à la température d'inversion.

Nous avons réalisé des mesures d'effet Hall diffé-

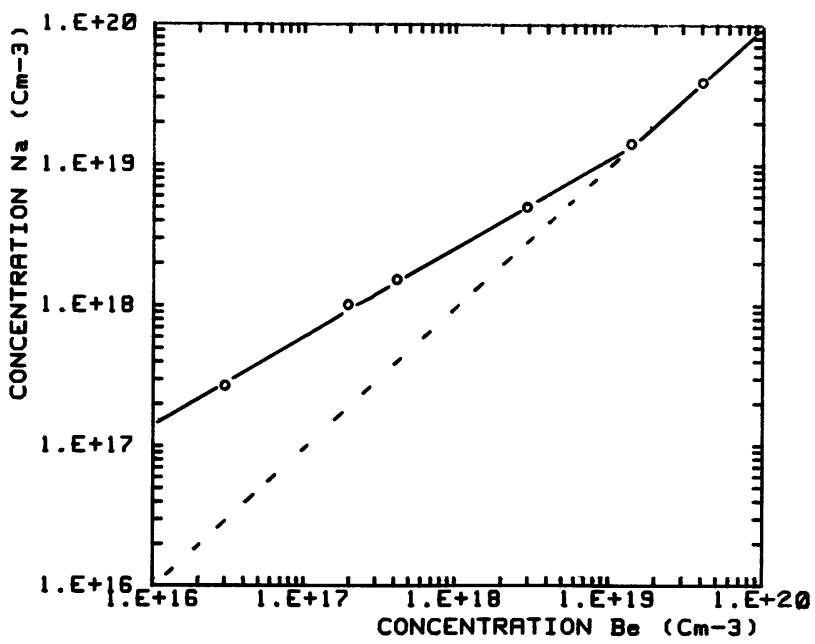

Fig. 5. - Concentrations d'accepteurs électriquement actifs déterminées à partir des mobilités globales à $77 \mathrm{~K}$ en fonction de la concentration de béryllium évaluée par SIMS.

[Electrical acceptors concentration deduced from mean Hall mobility at $77 \mathrm{~K}$ versus SIMS beryllium concentration.]

rentiel sur certains échantillons et nous avons constaté qu'il pouvait exister :

- une couche $\mathrm{N}$ en surface suivie d'une couche $\mathrm{P}$ pour des échantillons $\mathrm{N}$ au sens de l'effet Hall (Fig. 6) ;

- une couche $\mathrm{P}$ suivie d'une couche $\mathrm{N}$ à l'interface couche substrat pour des échantillons de type $P$ et une mobilité différentielle dans la zone $P$, supérieure à la mobilité globale mesurée (Fig. 7).

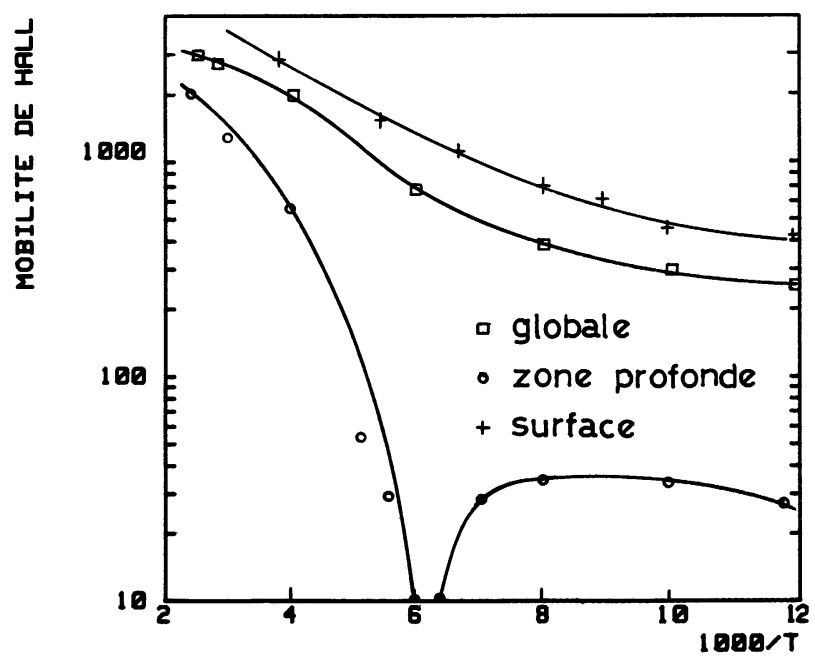

Fig. 6. - Mobilité de Hall d'un échantillon de type $\mathrm{N}$ en fonction de la température : ( $\square$ ) valeur moyenne de la couche; (O) valeur moyenne après attaque de $3000 \AA$; (+) mobilité de Hall différentielle.

[Hall mobility of a $\mathrm{N}$ type sample versus temperature : ( $\square$ ) mean value of the layer ; (O) mean value after $3000 \AA$ layer removal ; $(+)$ differential Hall mobility.] 


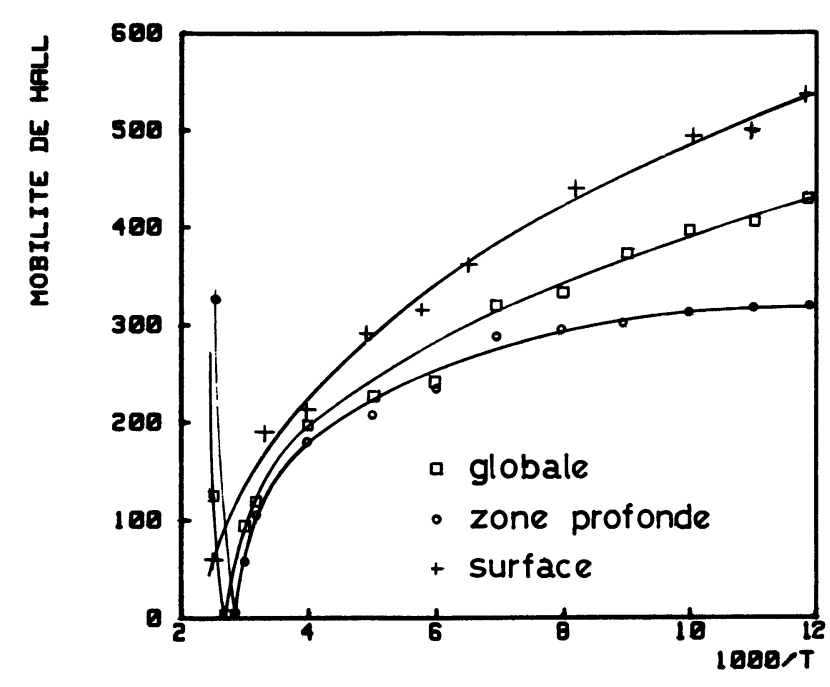

Fig. 7. - Mobilité de Hall d'un échantillon de type $\mathrm{P}$ en fonction de la température : ( $\square$ ) valeur moyenne de la couche; (O) valeur moyenne après attaque de $5000 \AA$; (+) mobilité de Hall différentielle.

[Hall mobility of a $\mathbf{P}$ type sample versus temperature : (ㅁ) mean value of the layer ; (O) mean value for $5000 \AA$ layer removal ; $(+)$ differential Hall mobility.]

\section{Interprétation des résultats.}

Une seule mesure de mobilité à $300 \mathrm{~K}$ des échantillons de type $\mathrm{P}$, conduit a priori à une compensation trop élevée par rapport aux concentrations SIMS. Mais les résultats précédents, nous amènent à formuler l'hypothèse d'une contribution d'électrons à la conductivité d'une couche de type $P$ avec une mobilité de Hall résultante plus faible que la mobilité réelle des trous, voire dans certains cas à une mobilité globale de type $\mathrm{N}$. Cette interprétation n'est possible qu'en considérant une superposition de deux couches de type $\mathrm{N}$ et $\mathrm{P}$, avec une transition graduelle de l'une à l'autre. L'oxygène assure le dopage de type $\mathbf{N}$ dans la partie de la couche où sa concentration excède celle du béryllium et joue le rôle de centre compensateur dans le restant de la couche.

Dans ce cas, la mobilité de Hall s'exprime en fonction des densités carrées ns et ps en électrons et en trous, et de leur mobilité correspondante $\mu_{n}$ et $\mu_{\mathrm{p}}[5,6]$

$$
\mu_{\mathrm{h}}=\frac{\mathrm{ps} \cdot \mu_{\mathrm{p}}^{2}-\mathrm{ns} \cdot \mu_{\mathrm{n}}^{2}}{\mathrm{ps} \cdot \mu_{\mathrm{p}}+\mathrm{ns} \cdot \mu_{\mathrm{n}}} .
$$

Compte tenu du rapport $b$ élevé des mobilités d'électrons par rapport à celle des trous $(b=25$ à $77 \mathrm{~K}$ ), une faible densité carrée de la couche de type $\mathrm{N}$, peut entraîner une réduction de mobilité mesurée, d'autant plus appréciable que la densité de béryllium est faible.
On peut expliquer ainsi le changement de type $\mathrm{P}$ à type $\mathrm{N}$ en fonction de la température : Stall et al. [1] et Loualiche et al. [8] ont montré que la concentration d'électrons d'une couche volontairement contaminée par de l'oxygène augmentait avec la température; dans le même temps l'efficacité des barrières de potentiel diminue lorsque la température augmente, ce qui permet une augmentation de la densité carrée d'électrons ns participant à la conduction. Ces effets induisent un changement de type pour les échantillons les moins dopés et une réduction de mobilité pour les autres.

Nous avons évalué ns pour les échantillons de type $P$ les moins dopés avec les hypothèses simplificatrices suivantes :

la contribution des électrons à la conductivité est faible par rapport à celle des trous et ns . $\mu_{\mathrm{n}}^{2}$ faible mais non négligeable devant ps . $\mu_{\mathrm{p}}^{2}$.

On obtient ainsi une expression approchée de la mobilité expérimentale

$$
\mu_{\mathrm{h}}=\mu_{\mathrm{p}}-\frac{\mathrm{ns} \cdot \mu_{\mathrm{n}}^{2}}{\mathrm{ps} \cdot \mu_{\mathrm{p}}} .
$$

Les mobilités $\mu_{\mathrm{n}}$ et $\mu_{\mathrm{p}}$ étant évaluées en considérant les concentrations SIMS de béryllium et d'oxygène pour la densité totale d'impuretés ionisées et la courbe $\mu_{\mathrm{h}}=f(\mathrm{Ni})$ à $77 \mathrm{~K}$ de la référence [2].

Les densités carrées d'électrons, évaluées à partir de la relation précédente, sont représentées sur la figure 8. Il existe une très forte corrélation entre la concentration électronique de la couche $\mathrm{N}$ et la concentration en oxygène.

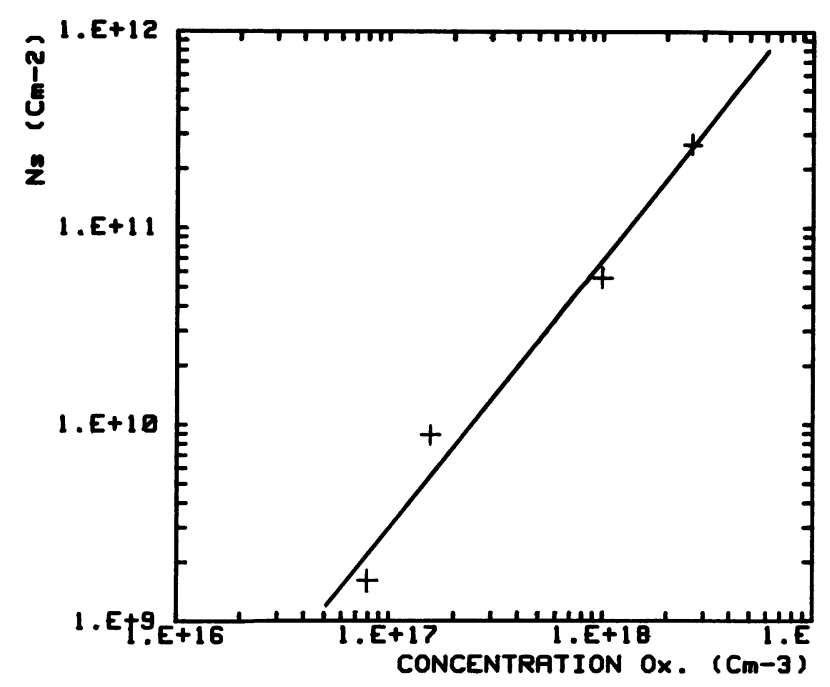

Fig. 8. - Concentration carrée d'électrons de la couche d'inversion en fonction de la concentration d'oxygène évaluée par SIMS.

[Square electron concentration of $\mathbf{N}$ type inversion layer versus SIMS oxygen concentration.] 


\section{Conclusion.}

L'activité électrique du béryllium dans les couches $\mathrm{In}_{x} \mathrm{Ga}_{1-x} \mathrm{As}$ déposées par épitaxie par jets moléculaires, est compensée par l'oxygène.

Les mesures d'effet Hall conduisent à une évaluation erronée de la compensation de la couche pour les plus faibles dopages du fait de l'existence d'une couche $\mathrm{N}$ à l'interface ou à la surface. Des mesures d'effet Hall différentiel ont confirmé l'existence de cette couche $\mathrm{N}$. Des mesures d'effet Hall en fonction de la température sont nécessaires pour mettre en évidence cet effet. La concentration en oxygène au démarrage de l'épitaxie, dépend de la qualité du vide résiduel du bâti d'épitaxie et du nettoyage du substrat [7] ; elle peut être supérieure à celle du béryllium, surtout pour les concentrations les plus faibles.

\section{Bibliographie}

[1] Stall, R., Wunda, R., Swaminathan, V., Appl. Phys. Let. 47 (1985) 518.

[2] Pearsall, T., Beuchet, G., Hirtz, J. P., Visentin, N., BONNET, M., RoIzes, A., Gallium Arsenide and Related Compounds, Vienne (1980).

[3] Tabattabie, K., Alavi, K., Markunas, R. J., FonstAD, C. G., J. Electrochem. Soc. 129 (1982) 2085.

[4] Wood, C. E., Desimone, D., Singer, K., Wicks, G. W., J. Appl. Phys. 53 (1982) 4230.
[5] Petritz, R., Phys. Rev. 110 (1958) 1254.

[6] Aina, L., Mattingly, M., Pende, K., Appl. Phys. Let. 49 (1986) 865.

[7] Lecorre, A., Caulet, J., Gauneau, M., LoualiChe, S., L'Haridon, H., LeCrosnier, D., Roizes, A., David, J. P., Appl. Phys. Let. 51 (1987) 1597.

[8] Loualiche, S., Gauneau, A., Lecorre, A., Lecrosnier, D., L'Haridon, H., Appl. Phys. Let. 51 (1987) 1361. 